\title{
A Note on Evolution Algebras as Banach Algebras
}

\author{
Rafael González-López, Juan Núñez-Valdés* \\ Department of Geometry and Topology, University of Seville, Spain \\ E-mail: jnvaldes@us.es
}

Received: 12 April 2021; Revised: 28 June 2021; Accepted: 30 June 2021

\begin{abstract}
With the objective of considering evolution algebras as Banach algebras, we show in this paper new results on normed evolution algebras obtained after the introduction of an evolution algebras operator, which generalizes the one introduced by Tian. The properties of this operator which let evolution algebras be endowed with a Banach space structure are shown.
\end{abstract}

Keywords: evolution algebras, normed algebras, Banach spaces, evolution operator, graphicable evolution algebras

MSC: 17D99, 46B20, 46B50

\section{Introduction}

Tian was the researcher who introduced evolution algebras, in his Ph.D. Thesis in 2004 [1]. Two years later, Vojtechovsky and himself published a paper on them [2], and finally, Tian published a book dealing extensively with these algebras in 2008 [3]. In that book, Tian showed several applications of these algebras, not only to different mathematical branches, as Algebra, Discrete Mathematics, and Statistics, for instance, but to other scientific disciplines, like Biology, Physics, or Engineering. Since those dates, evolution algebras have been deeply studied, respectively.

At present, there exist also several papers in the literature linking these algebras with different objects characteristic of other disciplines, like graphs, combinatorial structures, Markov chains and algebraic varieties, for instance. For examples, papers [4-7] and [8] can be cited. See also [9-10] and [11] for analysis concerning dynamical systems modeling chemotaxis and moment problems, respectively.

This paper continues with this last research line cited and tries to endow evolutions algebras with a norm. From a scientific point of view, this idea can be considered novel because there is practically no approach for the evolution algebras as Banach algebras and this subject has had no continuity in the form of papers, unlike what has happened with other types of algebras, such as Lie algebras, for instance (see [12]). Moreover, all results obtained could be considered a valuable starting point for a more in-depth study of this topic in Functional Analysis.

Let us recall that an $n$-dimensional evolution algebra is that algebra in which the relationships between its generators $\left\{e_{1}, \ldots, e_{n}\right\}$ are given by

Copyright (C2021 Juan Núñez-Valdés, et al.

DOI: https://doi.org/10.37256/cm.232021868

This is an open-access article distributed under a CC BY license

(Creative Commons Attribution 4.0 International License)

https://creativecommons.org/licenses/by/4.0/ 


$$
\left\{\begin{array}{l}
e_{i} \cdot e_{j}=0, \forall i \neq j, 1 \leq i, j \leq n \\
e_{i} \cdot e_{i}=\sum_{j=1}^{n} a_{j i} e_{j} .1 \leq i \leq n
\end{array}\right.
$$

Graphicable algebras, which were also introduced by Tian in [3], constitute a particular subclass of evolution algebras. They are defined as follows: an $n$-dimensional graphicable evolution algebra is a commutative, nonassociative algebra, with a set of generators $V=\left\{e_{1}, e_{2}, \ldots, e_{n}\right\}$ endowed with relations

$$
\left\{\begin{array}{l}
e_{i} \cdot e_{i}=e_{i}^{2}=\sum_{k=1}^{n} a_{k i} e_{k}, 1 \leq i \leq n, a_{k i} \in\{0,1\} \\
e_{i} \cdot e_{j}=0, i \neq j, 1 \leq i, j \leq n
\end{array}\right.
$$

Thus, it is obvious that a graphicable algebra is an evolution algebra, although the converse is not true in general.

Moving on now to Graph Theory, the most basic concepts, as simple graph, vertices, edges, adjacency, incidence, and adjacency matrix are assumed to be known ([13] can be consulted for details). Regarding the concept of the graph, Tian showed in [3] how to associate a given graph with an evolution algebra. He gave the following

Definition 1.1 Let $G=(V, E)$ be a graph, $V$ be the set of vertices of $G, E$ be the set of edges of $G$. We define an algebra $A(G)=\langle V \mid R\rangle$ as follows: taking $V=\left\{e_{1}, e_{2}, \ldots, e_{r}\right\}$ as the generator set and

$$
R=\left\{e_{i}^{2}=\sum_{e_{k} \in \Gamma\left(e_{i}\right)} e_{k} ; e_{i} \cdot e_{j}=0, \begin{array}{c}
i \neq j \\
1 \leq i, j \leq r
\end{array}\right\}
$$

as the set of defining relations, where $\Gamma\left(e_{i}\right)$ is the set of neighbours of $e_{i}$.

The algebra $A(G)$ is an evolution algebra as it can be checked straightforwardly. However, for the converse, there is no definition in [3] of the graph associated with an evolution algebra $A$. That association was introduced by Núñez, Silvero and Villar in [8] as follows

Definition 1.2 Given an $n$-dimensional evolution algebra $A$, the matrix associated with $A$ is that matrix $L(A)$ whose elements are, in columns, the coefficients of the fields $e_{j}$ of the basis of $A$ in the expressions of the brackets $e_{j}^{2}$ for $1 \leq j \leq n$.

From this last definition, a given evolution algebra $A$ has associated a weighted directed graph, possibly with loops, $G(A)=(V, E)$, as follows: $V$ is the set of generators of the algebra and $E$ is the set of edges linking $e_{i}$ with vertices in $\Gamma\left(e_{i}\right)$ for each $e_{i}$. The weight of each edge represents the coefficient in the corresponding bracket in the law of the algebra. Moreover, in the particular case of graphicable algebras, all of these weights are equal to 1 and they are omitted in the graph. Therefore, for a graphicable algebra, $A$, the associated graph to $A, G(A)$, has a binary adjacency matrix.

\section{Previous results on normed evolution algebras}

With the objective of describing the evolution flow quantitatively in an evolution algebra, Tian defined a norm on these algebras in [3].

Indeed, if $E$ is an evolution algebra with a generator set $\left\{e_{i} \mid i \in \Lambda\right\}$ over a field $\mathbb{K}$ (it can be supposed that $\mathbb{K}=\mathbb{R}$ without loss of generality), Tian defined a norm as follows.

In the first place, he considered the function $N$ from $E$ to the underlying field $\mathbb{K}$ given by

$$
N: E \mapsto \mathbb{R}, N(x)=\sum_{i}\left|a_{i}\right|
$$

for all element $x$ of the evolution algebra $E$, expressed as $x=\Sigma_{i} a_{i} e_{i}$. 
Then, he proved that this function was a norm because it verified the properties of non-negativity: $N(x) \geq 0$ and $N(x)=0$ if and only if $x=0$, linearity: $N(a x)=|a| N(x)$, for all $a \in \mathbb{K}$ and triangle inequality: $N(x+y)=N(x)+N(y)$, for all $x, y \in E$.

Therefore, an evolution algebra is a normed algebra. He denoted $N(x)=\|x\|$ and obtained some results on this function $N(x)$, which can be checked in [3].

Next, with the objective of getting some advances in this topic, we define in the next section an evolution algebras operator and we use it to endow these algebras with a new norm.

\section{A new evolution algebras operator}

In this section, we define a novel evolution operator of evolution algebras and we obtain its main properties.

Definition 3.1 Let $E$ be an evolution algebra. The operator $N w(E)$ is defined as

$$
N w(E)=\sum_{i \in \Lambda} N\left(e_{i}^{2}\right)
$$

Starting from this definition and by using techniques similar to others that have proven to be efficient (see [15-17], for instance), the following properties of this operator are obtained.

Proposition 3.2 Let $E$ be an evolution algebra whose structure constant are nonnegative integer and $G$ its associated graph (may be a multigraph). Then,

$$
N w(E)=|A(G)|
$$

Proof. Since $E$ is graphicable, $N\left(e_{i}\right)$ represents the numbers of vertices adjacent with the generator $e_{i}$. Then, $N\left(e_{i}\right)$ $=\delta\left(v_{i}\right)$ where $\delta\left(v_{i}\right)$ is the number of edges adjacent to $v_{i}$ and $v_{i}$ is the induced vertex of the graph $G$ by $e_{i}$. Now, result (2) is an immediate consequence of the fact that in a direct graph $G=(V, A)$ (or multigraph) it is verified that $\Sigma_{v \in V} \delta(v)$ $=|A(G)|$ (let us recall that the first fundamental theorem of Graph Theory states that the sum of the degrees of all its vertices coincides with twice its size, that is, with twice its number of edges, while if the graph is directed, then the double word is canceled in the statement, and the same occurs in multigraphs [13]).

Note 3.3 It is important to distinguish between the $\delta\left(e_{i}\right)$ of evolution algebras, which represents the number of vertices adjacent to $e_{i}$ and the $\delta(v)$ in a directed graph, which is the number of edges adjacent to $v$. It is easy to see that both notions coincide in a graphicable evolution algebra, since the corresponding graph is simple.

Proposition 3.4 Let $E$ be an $n$-dimensional evolution algebra. Then

$$
N w(E) \leq \frac{h n(n+1)}{2}
$$

is an upper bound of $N(E)$, where $a_{i j}$ are the structure constants of $E$, where $h=\max _{i j}\left|a_{i j}\right|$.

Proof. Let us denote $\bar{E}$ the evolution algebra whose structure constants are $b_{i j}=h$, if $a_{i j} \neq 0$ and 0 otherwise. Let us also denote by $B(\bar{E})$ to the skeleton algebra of $\bar{E}$ and by $G$ its associated graph (recall that according to Theorem 15 of Chapter 3 in [3], every evolution algebra $E$ can be reduced to a unique evolution algebra $E_{r}$ such that its evolution subalgebras in its hierarchy are all one-dimensional subalgebras. Tian calls such a unique evolution algebra $E_{r}$ the skeleton of $E$.)

Proposition 3.2 implies that 


$$
\begin{aligned}
N w(E) & =\sum_{i=1}^{n} N\left(e_{i}^{2}\right)=\sum_{i, j=1}^{n}\left|a_{i j}\right| \leq \sum_{i, j=1}^{n}\left|b_{i j}\right|=N w(\bar{E}) \\
& =h N w(B(\bar{E}))=2 h|A(G(B(\bar{E})))| \leq \frac{h n(n+1)}{2} .
\end{aligned}
$$

It completes the proof.

\section{The operator $N w(E)$ is a norm}

Definition 4.1 Let $V$ be a $\mathbb{K}$ vector space and $\left\{e_{1}, \ldots, e_{n}, \ldots\right\}$ be a set ofgenerators of $V$. over $V$.

Let us call $\mathcal{A} \mathcal{E}(V)$ to the space of all possible evolution algebras which can be defined by the laws $e_{i}^{2}=\sum_{k} a_{i k} e_{k}$
.

In this space we define the following operations. Let $E, \bar{E}$ be evolution algebras and $\lambda \in \mathbb{K}$ :

- $E+\bar{E}$ is the evolution algebra defined by the structure constants $b_{i k}$ such that $b_{i k}=a_{i k}+\bar{a}_{i k}$.

- $\lambda E$ is the evolution algebra defined by the structure constants $b_{i k}=\lambda a_{i k}$.

Note that it is very easy to check that $\mathcal{A E}(V)$ is a vector space with respect to both operations.

Proposition $4.2(\mathcal{A E}(V),+, \cdot)$ is a normed vector space under $N w(\cdot)$.

Proof. Let $E \in \mathcal{A} \mathcal{E}(V)$ be an evolution algebra defined by the laws $e_{i}^{2}=\sum_{k} a_{i k} e_{k}$. Then

$$
N w(E)=\sum_{i} N\left(e_{i}\right)=\sum_{i} \sum_{k}\left|a_{i k}\right|=\sum_{i, k}\left|a_{i k}\right| \geq 0
$$

and the equality is only reached if and only if $\left|a_{i k}\right|=0$, for all $i, k$.

Let us consider $\lambda \in \mathbb{K}$. We have that

$$
N w(\lambda E)=\sum_{i, k}\left|\lambda a_{i k}\right|=\sum_{i, k}|\lambda|\left|a_{i k}\right|=|\lambda| \sum_{i, k}\left|a_{i k}\right|=|\lambda| N w(E) .
$$

Let now $\bar{E} \in \mathcal{A} \mathcal{E}(V)$ be given by the products $e_{i}^{2}=\sum_{k} b_{i k} e_{k}$. Then, we have

$$
N w(E+\bar{E})=\sum_{i, k}\left|a_{i k}+b_{i k}\right| \leq \sum_{i, k}\left|a_{i k}\right|+\left|b_{i k}\right|=N w(E)+N w(\bar{E}) .
$$

It completes the proof.

Proposition 4.3 If $V$ is an $\mathbb{R}$-vector space of finite dimension, then $(\mathcal{A E}(V), N w(\cdot))$ is a Banach space.

Proof. Since $V$ is of finite dimension, then $\mathcal{A E}(V)$ is also of finite dimension and is defined over $\mathbb{R}$. So, $\mathcal{A} \mathcal{E}(V)$ is complete under its norm.

\section{Certain conclusions}

As we pointed out in the introduction, in this paper we have introduced an algebras operator, which generalizes the evolution operator introduced by Tian. Some properties of this new operator have been obtained and it allows us to consider evolution algebras as Banach algebras.

Indeed, through the previous section of the paper we have defined sets and operations, which, joint with this new 
operator $N w$, form a Banach space. These results confirm and increase the ones by Mellon and Velasco, who recently proved that every evolution algebra is a normed algebra, for a determined norm defined in terms of a fixed natural basis. They gave a necessary and sufficient condition for a normed evolution algebra to be Banach and showed that every non-degenerate Banach evolution algebra is finite-dimensional [14]. Note that, in general, the completion of a normed evolution algebra is not an evolution algebra.

As problems which remain open in this study, authors think, in the first place, that the results here shown, taken over $\mathbb{R}$, could be extended in the future over other fields in which evolution algebras could be defined.

Apart from that and although it was already implicitly used when Tian proved that any evolution operator is a bounded linear operator (see [3]), it is noteworthy how in a graph-icable evolution algebra (a concept that we have intuitively extended when the structure constants are non-negative integers) $N\left(e_{i}\right)^{2}$ coincides with the number of adjacent edges of $e_{i}$ or of the corresponding vertex $e_{i}$ in the graph that it induces. As a second possibility, it would be appropriate that this research could be continued to find results that connect the algebraic properties of evolution algebras with those of the graphs that they induce.

\section{Aknowledgement}

This paper is partially supported by national projects MTM2015-65397-PFEDER and autonomic P.A.I. Group FQM-326.

\section{References}

[1] Tian JP. Evolution algebra theory. Thesis (Ph.D.). University of California, Riverside; 2004. p. 145.

[2] Tian JP, Vojtechovsky P. Mathematical concepts of evolution algebras in non-mendelian genetics. Quasigroups Related Systems. 2006; 14(1): 111-122.

[3] Tian JP. Evolution algebras and their applications. Lecture Notes in Mathematics 1921. Berlin: Springer-Verlag; 2008.

[4] Cabrera Y, Siles M, Velasco MV. Evolution algebras of arbitrary dimension and their decompositions. Linear Algebra and its Applications. 2016; 495: 122-162.

[5] Elduque A, Labra A. Evolution algebras and graphs. Journal of Algebra and Its Applications. 2015; 14(7): 10.

[6] Lopez-Blazquez F, Nunez J, Recacha S, Villar MT. Evolution alge-bras, Graph Theory and Markov chains. 2020. Available from: arXiv:submit/3577568 [Accesed 24th June 2021].

[7] Nunez J, Rodriguez-Arevalo ML, Villar MT. Certain particular families of graph-icable algebras. Applied Mathematics and Computation. 2014; 246(1): 416-425.

[8] Nunez J, Silvero M, Villar MT, Mathematical tools for the future: Graph Theory and graphicable algebras. Applied Mathematics and Computation. 2013; 219(11): 6113-6125.

[9] Li T, Viglialoro G. Boundedness for a nonlocal reaction chemotaxis model even in the attraction-dominated regime. Differential Integral Equations. 2021; 34(5-6): 315-336.

[10] Viglialoro G, Woolley TE. Solvability of a Keller-Segel system with signaldependent sensitivity and essentially sublinear production. Applicable Analysis. 2020; 99(14): 2507-2525.

[11] Ghasemi M, Infusino M, Kuhlmann S, Marshall M. Moment problem for symmetric algebras of locally convex spaces. Integral Equations Operator Theory. 2018; 90(3): 1-19.

[12] Dynkin EB. Normed Lie algebras and analytic groups. American Mathematical Society Translation Series. 1953.

[13] Harary F. Graph Theory. Massachusetts: Reading; 1969.

[14] Mellon P, Velasco MV. Analytic aspects of evolution algebras. Banach Journal of Mathematical Analysis. 2019; 13(1): 113-132.

[15] Marin M. Generalized solutions in elasticity of micropolar bodies with voids. Folia Canariensis Academiae Scientiarum. 1996; 8(1): 101-106.

[16] Marin M. An uniqueness result for body with voids in linear thermoelasticity. Reports on Mathematics and its applications. 1997; 17(7): 103-113.

[17] Marin M. On the domain of influence in thermoelasticity of bodies with voids. Archivum Mathematicum. 1997; 33(4): 301-308. 